\title{
A SHORT-TIME PRONY METHOD FOR THE DETECTION OF TRANSIENTS
}

\author{
Annie Cuyt ${ }^{1}$, Wen-shin Lee ${ }^{1}$, and Min-nan Tsai ${ }^{1,2}$ \\ ${ }^{1}$ Department of Mathematics and Computer Science \\ University of Antwerp (CMI), Belgium \\ e-mail: \{annie.cuyt, wen-shin.lee\}@ unantwerpen.be \\ ${ }^{2}$ Department of Computer Science and Information Engineering \\ National Taiwan Normal University, Taiwan \\ e-mail: edwen0224@gmail.com
}

Keywords: Exponential analysis, sparse interpolation, Prony's method, transients, power system.

\begin{abstract}
Many parametric spectral methods are based on the classical algorithm of the French engineer G. de Prony for exponential analysis. A drawback of this method is that it cannot take into consideration any discontinuities due to the starting and ending of the exponential components at different instants.

We introduce a short-time Prony method that allows to extract the characteristics from such a signal and we illustrate the new method on a number of power system signals. All parameters in the signals can be extracted with high accuracy and we show how to monitor the occurrence of the transients dynamically.
\end{abstract}




\section{INTRODUCTION}

A transient is used to refer to any signal or wave that is short lived. Transient detection has applications in many highly dynamic signals such as power line analysis, speech and image processing, turbulent flow applications, to name just a few. In a power system signal, transients are observed as short lived high-frequency oscillations superimposed on the voltages or currents of the fundamental frequency which is $50 / 60 \mathrm{~Hz}$, as well as exponential components. They can be caused by lightnings, equipment faults, switching operations and so on.

Transient detection is a relatively common problem in many applications. They are often sinusoidal in nature and a lot of research has gone into the automation of their detection. We analyze how a sparsity based method can contribute to the power system analysis. A drawback of the standard Prony method for exponential analysis, is that it does not take into consideration the discontinuities due to the switching, in other words due to the exponential components to start and end at different instants.

In Section 2 the standard Prony method is recalled, while in Section 3 a short-time version of it is developed. The latter is applied successfully to a number of power signal simulations in Section 4. We use a damped sinusoidal model that is related to the phenomena typically observed at power system plants.

\section{THE STANDARD PRONY METHOD}

Let $\psi_{i}, \omega_{i}, \beta_{i}$ and $\gamma_{i}$ respectively denote the damping, frequency, amplitude and phase in each component of the signal

$$
\Phi(t)=\sum_{i=1}^{n} \alpha_{i} \exp \left(\phi_{i} t\right), \quad \alpha_{i}=\beta_{i} \exp \left(\text { i } \gamma_{i}\right), \quad \phi_{i}=\psi_{i}+\text { i } 2 \pi \omega_{i} .
$$

Let us assume that the frequency content in (1) is limited by

$$
\left|\Im\left(\phi_{i}\right) /(2 \pi)\right|=\left|\omega_{i}\right|<\Omega / 2, \quad i=1, \ldots, n,
$$

as required by the Shannon-Nyquist theorem, and let $\Phi(t)$ be sampled at the equidistant points $t_{j}=j \Delta$ for $j=0,1, \ldots, 2 n-1, \ldots$ with $\Delta \leq 1 / \Omega$. In the sequel we denote

$$
f_{j}:=\Phi\left(t_{j}\right), \quad j=0,1, \ldots, 2 n-1 .
$$

The aim now is to find $n, \phi_{1}, \ldots, \phi_{n}, \alpha_{1}, \ldots, \alpha_{n}$ from the measurements $f_{0}, \ldots, f_{2 n-1}, f_{2 n}, \ldots$ and the form (1) of the model for $\Phi(t)$. The inherent structure present in (1) allows to separate the computation of the nonlinear parameters $\phi_{i}$ from that of the linear parameters $\alpha_{i}$, as already dsicovered by the French engineer de Prony [7].

Let us define the Hankel matrices

$$
H_{n}^{(r)}:=\left[\begin{array}{ccc}
f_{r} & \cdots & f_{r+n-1} \\
\vdots & \ddots & \vdots \\
f_{r+n-1} & \cdots & f_{r+2 n-2}
\end{array}\right], \quad r \geq 0
$$

It is known [3, p. 603] that

$$
\operatorname{det} H_{\nu}^{(r)}=0, \quad \nu>n,
$$


and it is proved in [5] that, in the absence of noise,

$$
\begin{aligned}
& \operatorname{det} H_{n}^{(r)} \neq 0, \\
& \operatorname{det} H_{\nu}^{(r)}=0 \text { only accidentally, } \quad \nu<n .
\end{aligned}
$$

From these statements the number of components $n$ can be obtained as the (numerical) rank of $H_{\nu}^{(r)}$ for $\nu>n$. In practice, when the signal $\Phi(t)$ is affected by noise, the numerical rank is determined as the number of singular values of $H_{\nu}^{(r)}$ that rise above the noise level. In (4) this means that one finds $n$ singular values clearly above the noise level and the remaining $\nu-n$ at or below the noise level.

We further denote

$$
\lambda_{i}:=\exp \left(\phi_{i} \Delta\right), \quad i=1, \ldots, n .
$$

The $\lambda_{i}$ can be retrieved [4] as the generalized eigenvalues of the problem

$$
H_{n}^{(1)} v_{i}=\lambda_{i} H_{n}^{(0)} v_{i}, \quad i=1, \ldots, n
$$

where $v_{i}$ are the generalized right eigenvectors. Then from the values $\lambda_{i}$, the $\phi_{i}$ can uniquely be retrieved because of the restriction $\left|\Im\left(\phi_{i} \Delta\right)\right|<\pi$.

To conclude, one finds the $\alpha_{i}$ from the interpolation conditions

$$
\sum_{i=1}^{n} \alpha_{i} \exp \left(\phi_{i} t_{j}\right)=f_{j}, \quad j=0, \ldots, 2 n-1,
$$

either by solving the system in the least squares sense in the presence of noise or by solving a subset of $n$ consecutive interpolation conditions in case of a noisefree $\Phi(t)$. Note that

$$
\exp \left(\phi_{i} t_{j}\right)=\lambda_{i}^{j}
$$

and that the coefficient matrix of (6) is therefore a Vandermonde matrix.

We now present a reformulation of the exponential analysis problem using tools from rational approximation theory [1].

With $f_{j}=\Phi\left(t_{j}\right)$ we define the noisefree

$$
F(t)=\sum_{j=0}^{\infty} f_{j} t^{j}
$$

Since

$$
f_{j}=\sum_{i=1}^{n} \alpha_{i} \exp \left(j \phi_{i} \Delta\right)=\sum_{i=1}^{n} \alpha_{i} \lambda_{i}^{j}
$$

we can rewrite

$$
F(t)=\sum_{i=1}^{n} \frac{\alpha_{i}}{1-t \lambda_{i}}
$$

So we see that $F(t)$ is a rational function of degree $n-1$ in the numerator and $n$ in the denominator, with poles $1 / \lambda_{i}$. From Padé approximation theory we know that the Padé approximant $r_{n-1, n}(t)$ to $F(t)$ of degree $n-1$ in the numerator and $n$ in the denominator, reconstructs $F(t)$, in other words

$$
r_{n-1, n}(t)=F(t) .
$$


The partial fraction decomposition (8) is related to the Laplace transform of the exponential model (1), which explains why this approach is known as the Padé-Laplace method. When the signal $\Phi(t)$ is noisy, then the samples equal $f_{j}+\epsilon_{j}$ instead of $f_{j}$ where $\epsilon_{j}$ denotes a noise term. Let us denote the noisy series by

$$
F(t)+\epsilon(t)=\sum_{j=0}^{\infty}\left(f_{j}+\epsilon_{j}\right) t^{j}
$$

It is clear that the Padé approximant $r_{n-1, n}(t)$ does not reconstruct $F(t)+\epsilon(t)$ now as the latter is not a rational function anymore. For a novel way to make use of the Padé-Laplace reformulation in this situation, we refer the reader to [2].

\section{A SHORT-TIME PRONY METHOD}

When the model (1) changes dynamically across the time window used to collect the samples $f_{j}$, due to the fact that exponential components are switched on or off during the sampling, then the standard Prony method or its Padé-Laplace reformulation cannot be applied. In this section we adapt the model to allow for the components to start and/or end during the time of observation, to

$$
\Psi(t)=\sum_{i=1}^{n} \alpha_{i} \exp \left(\phi_{i}(t)\right)\left(u\left(t-s_{i}\right)-u\left(t-e_{i}\right)\right),
$$

where $s_{i}$ and $e_{i}$ are the start and end times of the $i$-th signal component and $u(t)$ is the unit step function. For ease of notation we still denote $f_{j}$ for the time sample at time $t_{j}=j \Delta$. From the context it will always be clear whether $f_{j}$ comes from $\Phi(t)$ as in (1) or $\Psi(t)$ as in (9). We also retain the notation $\lambda_{i}=\exp \left(\phi_{i} \Delta\right), i=1, \ldots, n$.

We now combine the sampling of (9) with a window function $w(j-r)$ that is only nonzero in the time interval $[r \Delta,(r+2 \nu-1) \Delta]$ with $\nu \geq n(r)$ where $n(r)$ denotes the number of exponential components switched on somewhere in the course of the time interval $[r \Delta,(r+$ $2 \nu-1) \Delta]$. Obviously $n(r) \leq n$. In our experiments we used the rectangular window function

$$
w(j-r)= \begin{cases}1, & r \leq j \leq r+2 \nu-1, \\ 0, & \text { elsewhere }\end{cases}
$$

The Hankel matrix $H_{\nu}^{(r)}$ as defined in (2), makes use of the samples $f_{r}, \ldots, f_{r+2 \nu-1}$ of (9). We consider its (numerical) rank stable when it does not alter while increasing $r$ to $r+\rho$ with $\rho>0$. Note that during the inspection of the (numerical) rank of the matrices $H_{\nu}^{(r)}, \ldots, H_{\nu}^{(r+\rho)}$, the early samples shift out of the matrix while they are being replaced by new samples. When we observe that the (numerical) rank of $H_{\nu}^{(r)}$ does not change in the time window $[r \Delta,(r+$ $\rho+2 \nu-1) \Delta]$, then the standard Prony method can be applied to extract the characteristics of the $n(r)$ components active in that time window. When the (numerical) rank of $H_{\nu}^{(r)}$ fluctuates while increasing $r$, we know that components are being switched on or off, and the extraction of their characteristics using Prony's method should be postponed to a stable time window because the signal is not following a model of type (1) in the observed window.

The width of the time window here is related to the number $n(r)$ of components in (9) that are switched on in the considered time interval. A smaller time window allows more easily to find stable intervals, while a larger time window allows to work with more components. This is very similar to a similar conclusion for the short-time Fourier transform, where a narrow 
window gives good time resolution but poor frequency resolution and a wide window gives better frequency resolution but poor time resolution.

Then some words on the numerical computation of the $\phi_{i}$ and $\alpha_{i}$ in practice, from noise corrupted samples $f_{j}$. When the largest time window of stability for the computation of $\nu$ generalized eigenvalues in total (comprising the $n(r)$ active ones representing the signal space and $\nu-n(r)$ additional ones modeling the noise space), is $[r \Delta,(r+\rho+2 \nu-1) \Delta]$, then the $\lambda_{i}=\exp \left(\phi_{i} \Delta\right)$ present in that time window are computed in the least squares sense rather than from (5),

$$
H_{\nu+\lfloor\rho / 2\rfloor, \nu}^{(r+1)} v_{i}=\lambda_{i} H_{\nu+\lfloor\rho / 2\rfloor, \nu}^{(r)} v_{i}, \quad i=1, \ldots, \nu,
$$

where the subscript of the Hankel structured matrix now indicates its dimension. Several popular exponential analysis implementations that can be used for this purpose are given in $[9,8,4]$.

To adapt the Padé-Laplace formulation to work with a window function, we consider

$$
F(t-r \Delta)=\sum_{i=1}^{n(r)} \frac{\alpha_{i}}{1-(t-r \Delta) \lambda_{i}}, \quad t_{r} \leq t \leq t_{r+\rho+2 n(r)-1} .
$$

Since this time interval delivers enough samples $f_{j}$ for the computation of the Padé approximant $r_{n(r)-1, n(r)}(t-r \Delta)$, namely at least $2 n(r)$, the reformulation still holds.

\section{SIMULATION RESULTS}

We describe some experiments that illustrate the use of the short-time Prony method. The considered synthesized electric signals are taken from [6]. Since these are a linear combination of switched cosine functions, more precisely

$$
\Psi(t)=\sum_{i=1}^{n / 2} \beta_{i} \cos \left(2 \pi \omega_{i}(t)+\gamma_{i}\right) \exp \left(\psi_{i}\left(t-s_{i}\right)\right)\left(u\left(t-s_{i}\right)-u\left(t-e_{i}\right)\right),
$$

each term is represented by 2 exponential terms with complex conjugate values $\phi_{i}$. The characteristics of the test signals are given in Table 1. Besides the start and end moments $s_{i}$ and $e_{i}$ of each component in seconds, we also list the sample numbers $S_{i}$ and $E_{i}$ at which the switch occurs. All signals are monitored for a total time span of 128 samples, numbered from 0 to 127. The sampling rate is given by $1 / \Delta$ (in $\mathrm{Hz}$ ) where $\Delta$ is the time step. Each signal is then corrupted by $32 \mathrm{~dB}$ white Gaussian noise, which for some test signals is quite a lot more than in [6]. After extracting the frequencies $\omega_{i}$ and damping factor $\psi_{i}$, the computed coefficient $\beta_{i} \exp \left(-\psi_{i} s_{i}\right)$ needs to be corrected using the $s_{i}$ obtained from the singular value plots, in order to have the correct amplitude $\beta_{i}$.

In Figure 1 the four test signals are graphed over the entire observation window. We remark that all signals are dead at $t=127 \Delta$, in other words $f_{127}=0$. For each noise corrupted signal we graph in Figure 2 the dynamic evolution of the numerical rank of a particular Hankel matrix by displaying how the $\nu$ singular values of $H_{\nu}^{(r)}$ change when $r$ is ranging from 0 to $129-2 \nu$ (the value of $r$ is on the $x$-axis). We display respectively the singular values of $H_{4}^{(r)}$ for $\Psi_{1}(t)$, $H_{6}^{(r)}$ for $\Psi_{2}(t), H_{6}^{(r)}$ for $\Psi_{3}(t)$ and $H_{8}^{(r)}$ for $\Psi_{4}(t)$, and this for $0 \leq r \leq 129-2 \nu$. Let us discuss these graphs before turning to the reconstruction of the signal parameters.

In $\Psi_{1}(t)$ the Hankel matrices $H_{4}^{(0)}$ to $H_{4}^{(30)}$ indicate a numerical rank of zero. Then there is a switch from no components at all to one component at sample 37 which enters the Hankel matrix $H_{4}^{(31)}$ for which neither statement (3) nor statement (4) hold. Nothing can be deduced 


\begin{tabular}{|c|c|c|c|c|c|c|c|c|c|c|}
\hline & $n$ & $\beta_{i}$ & $\gamma_{i}$ & $\omega_{i}$ & $\psi_{i}$ & $s_{i}$ & $S_{i}$ & $e_{i}$ & $E_{i}$ & $1 / \Delta$ \\
\hline$\Psi_{1}(t)$ & 1 & 1.000 & $-\pi / 2$ & 480 & -0.080 & 0.0308 & 37 & 0.1059 & 127 & 1200 \\
\hline$\Psi_{2}(t)$ & 3 & 1.000 & $-\pi / 2$ & 60 & 0.000 & 0.0000 & 0 & 0.0308 & 37 & 1200 \\
& & 1.000 & $3 \pi / 4$ & 60 & 0.000 & 0.0308 & 37 & 0.0625 & 75 & \\
& & 1.000 & $-\pi / 2$ & 60 & 0.000 & 0.0625 & 75 & 0.1059 & 127 & \\
\hline$\Psi_{3}(t)$ & 3 & 1.000 & 0 & 50 & 0.000 & 0.0000 & 0 & 0.0200 & 30 & 1500 \\
& & 0.250 & $\pi / 2$ & 100 & -0.100 & 0.0200 & 30 & 0.0533 & 80 & \\
& & 0.100 & $38 \pi / 100$ & 300 & 0.030 & 0.0447 & 67 & 0.0847 & 127 & \\
\hline$\Psi_{4}(t)$ & 4 & 1.000 & 0 & 60 & 0.000 & 0.0000 & 0 & 0.0333 & 40 & 1200 \\
& & 0.500 & $\pi / 2$ & 60 & 0.000 & 0.0333 & 40 & 0.0917 & 110 & \\
& & 0.050 & $-37 \pi / 100$ & 180 & 0.000 & 0.0417 & 50 & 0.0833 & 100 & \\
& & 0.200 & $-\pi / 2$ & 360 & -0.100 & 0.0500 & 60 & 0.1059 & 127 & \\
\hline
\end{tabular}

Table 1: Signal parameters for the four test signals.
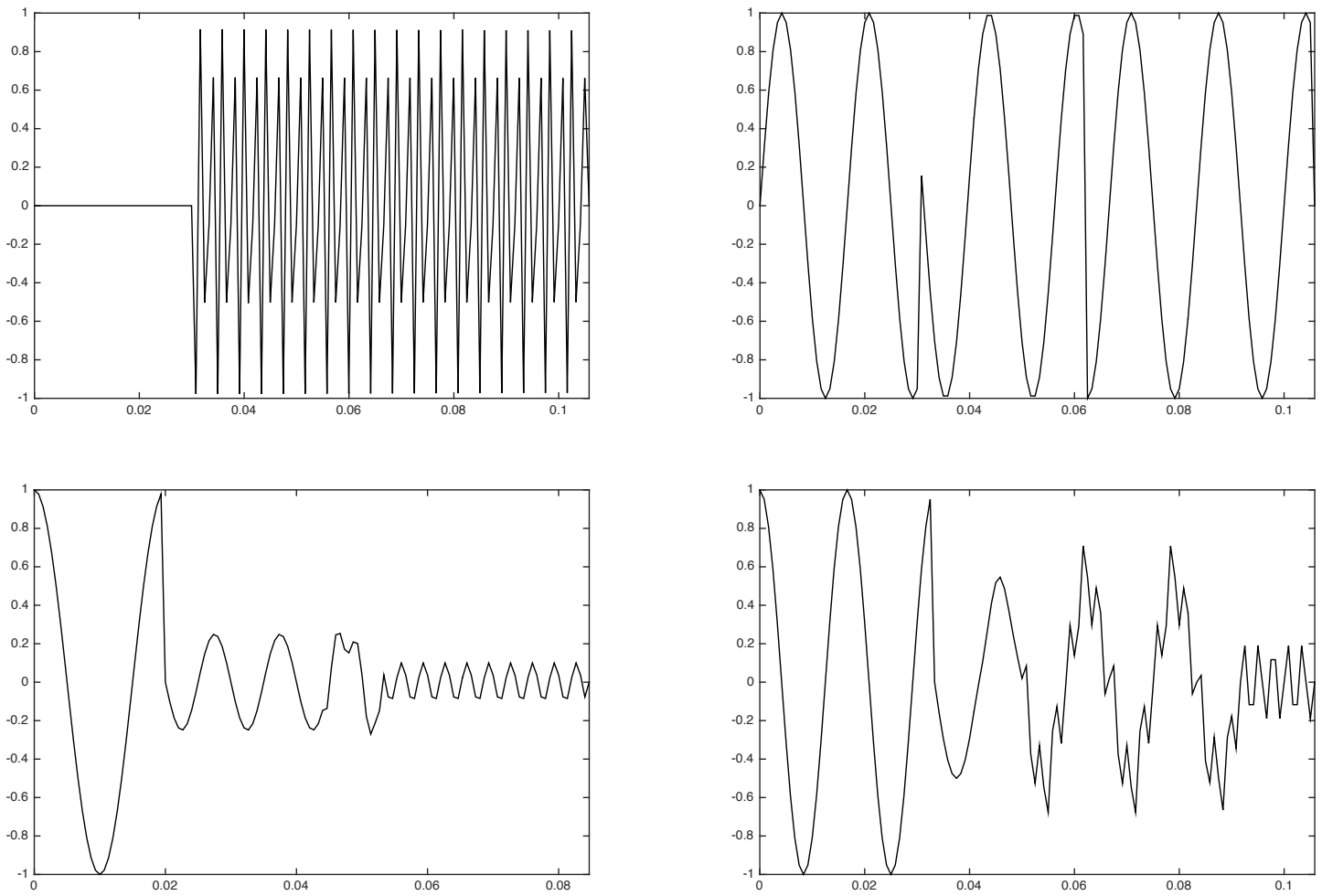

Figure 1: The signals $\Psi_{1}(t)$ (top left), $\Psi_{2}(t)$ (top right), $\Psi_{3}(t)$ (bottom left), $\Psi_{4}(t)$ (bottom right). 

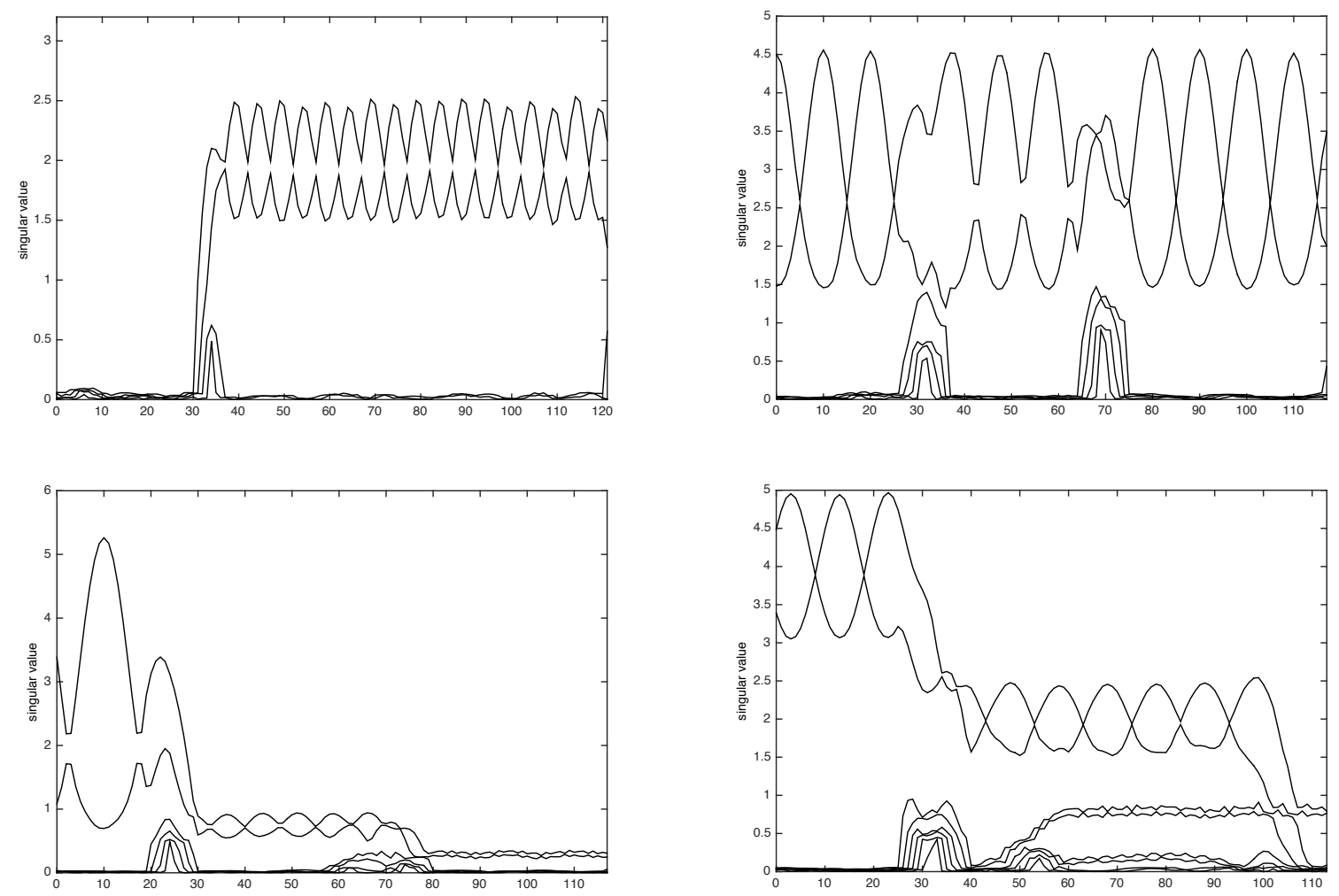

Figure 2: The dynamic singular value plots for $\Psi_{1}(t)$ (top left), $\Psi_{2}(t)$ (top right), $\Psi_{3}(t)$ (bottom left), $\Psi_{4}(t)$ (bottom right).

from the singular value behaviour to $H_{4}^{(36)}$. From $H_{4}^{(37)}$ on only one component is present and this for the remainder of the interval. The numerical rank is stable and equal to 2 . The single component can be identified from the signal samples $f_{r}, 37 \leq r \leq 126$.

In $\Psi_{2}(t)$ three different components show up, but consecutively: the switches happen at the time samples indexed 37, 75 and 127. So the numerical rank of the observed Hankel matrix is unstable while samples from different signals move out and in because the conclusions (3) and (4) do not hold during the short time span of the instability. To be more precise: sample 37 enters $H_{6}^{(r)}$ for $r+10=37$, sample 75 enters $H_{6}^{(r)}$ for $r+10=75$ and sample 127 enters $H_{6}^{(r)}$ for $r+10=127$. So the numerical rank instability is observed for $27 \leq r<37,65 \leq r<75$ and at $r=117$. Except for these unstable rank windows, the numerical rank equals 2. Each of the components can be identified separately: the first from $H_{\nu}^{(r)}$ with $0 \leq r$ and $r+2 \nu-2 \leq 36$, the second one from $H_{\nu}^{(r)}$ with $37 \leq r$ and $r+2 \nu-2 \leq 74$, the third one from $H_{\nu}^{(r)}$ with $75 \leq r$ and $r+2 \nu-2 \leq 126$.

The results for $\Psi_{3}(t)$ and $\Psi_{4}(t)$ are more interesting. In $\Psi_{3}(t)$ the exponential model (1) is interrupted from $H_{6}^{(20)}$ to $H_{6}^{(29)}$ for a first time. But the first exponential component can be computed from the samples 0 to 29 . Likewise the second component can be computed from the samples 30 to 66 . In $H_{6}^{(57)}$ sample 67 sneaks in. From $H_{6}^{(67)}$ to $H_{6}^{(69)}$ components two and three strictly follow (1) but this window involving the samples 67 to 79 is barely enough to allow their identification. However, exponential component three can be computed from the samples 80 to 126.

In $\Psi_{4}(t)$ a similar situation arises, but now there is a sufficiently large window of stability, 


\begin{tabular}{|c|c|c|c|c|c|}
\hline & $n$ & $\beta_{i}$ & $\gamma_{i}$ & $\omega_{i}$ & $\psi_{i}$ \\
\hline$\Psi_{1}(t)$ & 1 & 1.006 & -1.565 & 479.99 & -0.250 \\
\hline$\Psi_{2}(t)$ & 3 & 1.013 & -1.570 & 60.05 & -0.784 \\
& & 1.013 & 2.339 & 60.05 & -0.764 \\
& & 1.016 & -1.597 & 60.05 & -0.735 \\
\hline$\Psi_{3}(t)$ & 3 & 1.004 & 0.015 & 50.02 & -0.377 \\
& & 0.257 & 1.563 & 100.04 & -2.347 \\
& & 0.117 & 1.224 & 299.92 & -6.417 \\
\hline$\Psi_{4}(t)$ & 4 & 1.006 & 0.009 & 60.03 & -0.391 \\
& & 0.503 & 1.557 & 60.04 & -0.319 \\
& & 0.058 & -1.613 & 181.08 & 10.250 \\
& & 0.202 & -1.567 & 360.00 & -0.519 \\
\hline
\end{tabular}

Table 2: Reconstructed signal parameters for the four test signals.

namely from $H_{8}^{(60)}$ to $H_{8}^{(85)}$ when $r+14=99$, to allow for the identification of the last three components while they strictly adhere to model (1). The numerical rank is stable and equal to 6 in that window. The remaining first component can be identified from the samples 0 to 39 since $H_{8}^{(r)}$ indicates a stable numerical rank of 2 for $0 \leq r \leq 25$.

All reconstructions of $\alpha_{i}$ and $\phi_{i}$ have been made from the largest stable time windows, as mentioned in (10) and can be found in Table 2. The displayed values are an average of reconstructions over a 100 different noise realizations. We remark that the damping factors $\psi_{i}$ are more noise sensitive than the other parameters.

\section{CONCLUSION}

We have introduced a short-time Prony method that allows to extract the characteristics from a signal in which the exponential components are switched on and off, a situation that the standard Prony method is unable to deal with. The new method was illustrated on a number of power system signals taken from [6]. All parameters in the sinusoidal model (9) could be extracted with high accuracy and the occurrence of the transient could be monitored from the dynamics of the singular values of particular Hankel matrices.

\section{REFERENCES}

[1] Z. Bajzer, A.C. Myers, S.S. Sedarous, and F.G. Prendergast. Padé-Laplace method for analysis of fluorescence intensity decay. Biophys J., 56(1):79-93, 1989.

[2] A. Cuyt, W.-s. Lee, G. Lo Bianco, and M.-n. Tsai. How to pick up faint components in exponential analysis. Technical report, Universiteit Antwerpen, 2016.

[3] P. Henrici. Applied and computational complex analysis I. John Wiley \& Sons, New York, 1974.

[4] Y. Hua and T.K. Sarkar. Matrix pencil method for estimating parameters of exponentially damped/undamped sinusoids in noise. IEEE Trans. Acoust. Speech Signal Process., 38:814-824, 1990. 
[5] E. Kaltofen, W.-s. Lee, and A.A. Lobo. Early termination in Ben-Or/Tiwari sparse interpolation and a hybrid of Zippel's algorithm. In Proceedings of the 2000 International Symposium on Symbolic and Algebraic Computation, 192-201, ACM Press, New York, NY, USA, 2000.

[6] L. Lovisolo, E.A.B. da Silva, M.A.M. Rodrigues, and P.S.R. Diniz. Efficient coherent adaptive representations of monitored electric signals in power systems using damped sinusoids. IEEE Trans. Signal Proc., 53(10):3831-3846, 2005.

[7] R. de Prony. Essai expérimental et analytique sur les lois de la dilatabilité des fluides élastiques et sur celles de la force expansive de la vapeur de l'eau et de la vapeur de l'alkool, à différentes températures. J. Ec. Poly., 1:24-76, 1795.

[8] R. Roy and T. Kailath. ESPRIT-estimation of signal parameters via rotational invariance techniques. IEEE Trans. Acoustics, Speech and Signal Proc., 37(7):984-995, 1989.

[9] R.O. Schmidt. Multiple emitter location and signal parameter estimation. IEEE Trans. Antennas and Propagation, AP-34(3):276-280, 1986. 